
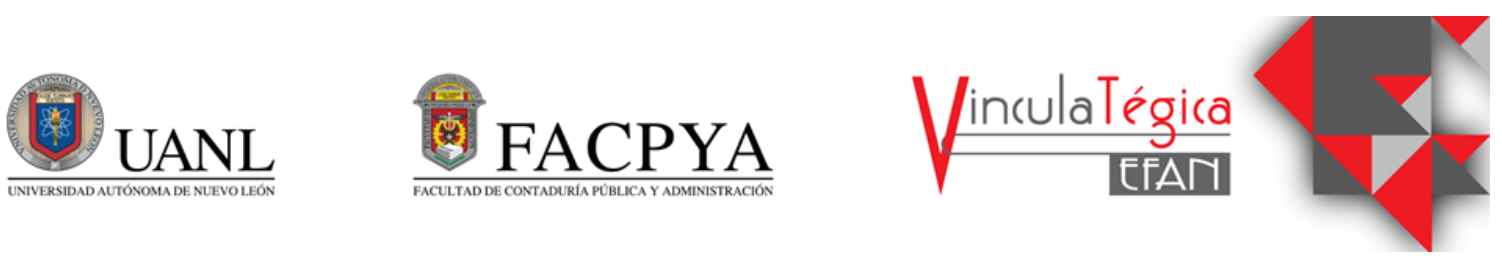

\title{
Flipgrid en la enseñanza aprendizaje y medio de interacción entre estudiantes de nivel superior durante la pandemia del Covid-19
}

\author{
Laura Alicia Hernández Moreno ${ }^{1}$, Juan Gabriel López Solórzano ${ }^{2}$ \\ y Celia Irma Villanueva $\mathrm{Chi}^{3}$
}

\author{
${ }^{1}$ Universidad Autónoma de Nuevo León; Facultad de Contaduría Pública y Administración, \\ laura.hernandezmr@uanl.edu.mx, Ciudad Universitaria San Nicolás de los Garza, Nuevo León, México. C.P. \\ 66451., Teléfono (81) 8329-4001 \\ ${ }^{2}$ Universidad Autónoma de Nuevo León; Facultad de Contaduría Pública y Administración, \\ juan.lopezsr@uanl.edu.mx, Ciudad Universitaria San Nicolás de los Garza, Nuevo León, México. C.P. \\ 66451., Teléfono (81) 8329-4001 \\ ${ }^{3}$ Universidad Autónoma de Nuevo León; Facultad de Contaduría Pública y Administración, \\ celia.villanuevach@uanl.edu.mx, Ciudad Universitaria San Nicolás de los Garza, Nuevo León, México. C.P. \\ 66451., Teléfono (81) 8329-4001)
}

Información del artículo revisado por pares

Fecha de aceptación: junio-2021

Fecha de publicación en línea: diciembre-2021

DOI: https://doi.org/10.29105/vtga7.2-70

\begin{abstract}
Resumen
El aprendizaje significativo y social es una tarea que hoy en día los docentes deben de cumplir y más a raíz de la pandemia covid-19. El objetivo del presente trabajo se expresa a través de la siguiente pregunta de investigación: ¿Cuál es la experiencia y satisfacción en el uso de la herramienta Flipgrid en estudiantes de la carrera de Licenciatura en Tecnologías de Información en una universidad pública del noreste de México, como recurso en su formación académica y medio de interacción en un ambiente virtual derivado de la pandemia? La metodología que se siguió, fue con alcance del tipo exploratorio descriptivo; con un enfoque del tipo mixto. Para el enfoque cuantitativo se diseñó y aplicó una encuesta compuesta por 8 preguntas cerradas categorizadas en una escala de Likert del 1 al 5. En el enfoque cualitativo se aplicó una metodología sistémica la cual consistió en cinco etapas: Planeación, desarrollo, obtención de los datos, análisis de los datos y evaluación. Los resultados indican un porcentaje promedio de satisfacción del $68 \%$ en el uso de la herramienta flipgrid, para el desarrollo de las actividades solicitadas a los estudiantes que participaron en
\end{abstract}

\begin{abstract}
Meaningful and social learning is a task that today's teachers must fulfill, especially in the wake of the covid19 pandemic. The objective of this work is expressed through the following research question: What is the experience and satisfaction in the use of the Flipgrid tool in students of the Bachelor's degree in Information Technology at a public university in northeastern Mexico, as a resource in their academic training and means of interaction in a virtual environment derived from the pandemic? The methodology followed was exploratory and descriptive, with a mixed approach. For the quantitative approach, a survey composed of 8 closed questions categorized on a Likert scale from 1 to 5 was designed and applied. In the qualitative approach, a systemic methodology was applied, consisting of five stages: planning, development, data collection, data analysis and evaluation. The results indicate an average satisfaction rate of $68 \%$ in the use of the flipgrid tool for the development of the activities requested of the students who participated in this study. Only 3\% reported having carried out some academic activity of this type, only with a different software. Considering the above, it is necessary for the teacher to try to be creative and innovative in the classroom, seeking to generate in
\end{abstract}


este estudio. Solo un $3 \%$ refiere haber realizado alguna actividad académica de este tipo, solo que con un software diferente. Considerando lo anterior, se hace necesario que el docente trate de ser creativo e innovador en el aula, buscando, el generar en el estudiante el conocimiento y habilidades que los estudiantes requieren en su ámbito académico y profesional.

Palabras clave: flipgrid, enseñanza aprendizaje, medio de interacción, pandemia covid-19, conocimiento y habilidades.

\section{INTRODUCCIÓN}

El uso de tecnologías es una de las habilidades que los profesores de este siglo deben de desarrollar para dar respuesta a las circunstancias que hoy en día están presentes en el proceso de enseñanzaaprendizaje. Estas tecnologías le permiten al profesor ser más innovador al crear diversas actividades que promuevan la formación, el trabajo colaborativo, la evaluación y feedback, esto favorece al estudiante en su aprendizaje y estimula la generación de su propio conocimiento (Walss,2021).

Mendoza y Vázquez (2020) afirman que a causa de la pandemia Covid 19 en este año, la educación se realiza de forma virtual, el empleo de la tecnología es más importante, y por consecuencia es fundamental plantear nuevas maneras de realizar el proceso educativo. La tecnología contribuye a que los estudiantes puedan ser más autónomos en la realización de sus actividades $\mathrm{y}$ analicen sobre su propio aprendizaje, mostrando su avance, dando a conocer a los demás participantes su conocimiento con soluciones creativas y como un medio de interacción entre aprender y socializar.

De todas las herramientas tecnológicas las que han ganado importancia son las llamadas tecnologías de respuesta por video, las cuales son herramientas sociales que permiten a las personas participar y colaborar con otros (Carrie Taylor \& Timothy Hinchman, 2020).

Siguiendo con Walss (2021) recomienda tecnologías que fomenten la evaluación educativa, que sean sin costo o de bajo costo que puedan integrarse a the student the knowledge and skills that students require in their academic and professional environment.

Key words: flipgrid, teaching learning, interaction medium, covid-19 pandemic, knowledge and skills. JEL: I2, D8, D83.

plataformas digitales didácticas, adaptables desde el nivel educativo básico hasta nivel superior como lo son: EDpuzzle, Flipgrid, Genially, Mentimeter, Nearpol, Quizlet, Piazza, Socrative, WebAssing y Wheeldecide.

Flipgrid es una herramienta de aprendizaje de Microsoft actualmente como recurso gratuito, en la que los estudiantes pueden interactuar con otros (estudiantes y profesor) ya que permite generar la discusión a través de un tema seleccionado por el profesor, incrementa la capacidad de expresión, permite elaborar tareas, grabar opiniones y respuestas de los alumnos en videos cortos, fortalece la seguridad en los estudiantes, el profesor obtiene un feedback casi inmediato sobre lo aprendido, estimula al estudiante a la construcción su propio conocimiento al participar estructurando su opinión y compartiendo con los demás (Murray, 2018). Además, propicia la colaboración y el aprendizaje social entre los estudiantes (Stoszkowski, 2018).

Las Universidades hoy en día tienen la necesidad de incluir tecnologías innovadoras en sus procesos de enseñanzaaprendizaje para realizar de manera efectiva actividades creativas que se adecúen a las circunstancias actuales, que fomente en el estudiante su atención y promueva un aprendizaje significativo (Mendoza y Vazquez,2020). Por otra parte, es muy importante considerar que en la formación virtual contar con una gran variedad de e-actividades es primordial para lograr una educación de calidad (Silva, 2017). Actualmente la importancia de la variedad de actividades no solo es para una formación virtual, sino para cualquier modalidad y en 
este sentido es que el profesor debe innovar.

Para que el aprendizaje sea

significativo, se deben incluir

actividades creativas que se adecuen al

En este contexto es importante el conocer cuál es la experiencia y satisfacción que los estudiantes de la carrera de Licenciatura en Tecnologías de Información en una universidad del noreste de México sobre el uso de la herramienta tecnológica Flipgrid para su formación académica y medio de interacción con sus compañeros y profesor en un ambiente educativo virtual. Se realizó esta investigación ya que se considera que esta herramienta fomenta el desarrollo de aprendizaje y de habilidades técnicas y blandas a través del diseño de imágenes, videos, audios, representa una forma de comunicar ideas, sentimientos y conocimientos. Es importante compartir la experiencia sobre su uso y que otros profesores la integren como un recurso de evaluación, y fomentar que las actividades desarrolladas por los estudiantes traten de ser más originales y como una forma de innovar en la práctica docente.

De manera concreta el objetivo sobre el cual versa el presente trabajo se expresa a través de la siguiente pregunta de investigación: ¿Cuál es la experiencia y satisfacción en el uso de la herramienta Flipgrid en estudiantes de la carrera de Licenciatura en Tecnologías de Información en una universidad del noreste de México, como recurso en su formación académica y medio de interacción en un ambiente virtual derivado de la pandemia?

La propuesta de investigación se considera justificable ya que, en los primeros meses del año 2020 el COVID-19 fue declarada pandemia por la Organización Mundial de la Salud (OMS), la forma de impartir la educación cambió de un día para otro. Las medidas tomadas por la Secretaría de Salud y la Secretaría de Educación en
México establecieron que todo el sistema educativo habría de suspender las clases presenciales para ser llevadas a cabo en forma virtual en donde el aprendizaje se realizaría fuertemente a través de la tecnología y esta se convertiría en el principal medio de comunicación y forma de dar cumplimiento a los programas educativos. Considerando lo anterior, los autores de este estudio definen a Flipgrid como una herramienta que por sus características se considera relevante su aplicación en la educación virtual e incluso en otras modalidades educativas, siendo una oportunidad el incorporarla y evaluar los beneficios que ofrece.

\section{MARCO TEÓRICO}

\subsection{Antecedentes}

La plataforma Flipgrid fue fundada en el año 2015 por el Dr. Charlie Miller, Jim Leslie y Phil Soran con la finalidad de cambiar la función del video en clase, utilizando esta herramienta para promover la interacción de los estudiantes. Flipgrid es utilizado por educadores desde nivel básico preescolar hasta el nivel superior de doctorado en 180 países. Flipgrid es aceptado por los estudiantes ya que les permite emitir su opinión con videos cortos, compartirlos y respetar los videos de los demás (Megiddo, 2018).

Flipgrid (https://info.flipgrid.com/) es una plataforma de discusión de video cortos gratuita de Microsoft que ayuda a los profesores a ver y escuchar a todos los estudiantes en clase y fomentar el aprendizaje social generando interés. En Flipgrid, los profesores publican un tema de discusión y los estudiantes contestan con videos cortos, pueden interactuar con sus compañeros comentando los videos y el docente mantiene el control total.

Una vez que el profesor crea una cuenta en https://info.flipgrid.com/ inicia con un tema que desea que desarrollen los alumnos para crear su video, posteriormente se elige como acceden los alumnos, lo cual puede ser por medio de un correo electrónico, asignando nombres de usuarios a cada uno o a través de un código de unión único y una contraseña. Finalmente se comparte el tema usando Google Classroom, Microsoft Teams o 
Remind, o proporcionando a los estudiantes el código de unión único que podrán utilizar a través de la app si se utiliza un dispositivo móvil o en el explorador de internet lo cual permitirá conectarse con la comunidad. Los alumnos después de ingresar su código inician sesión, comparten grabando un video corto con acceso a todo lo que necesitan para contar su historia o realizar su actividad, incluidos texto, emojis, entintado, pizarrones, grabación de pantalla, la capacidad de cargar archivos y permite el uso de rubricas de evaluación.

Gosse (2019) afirma que Flipgrid se organiza con tableros que se dividen en varios temas, de esa manera el estudiante identifica en donde va a entregar el video que se le solicitó. Los tableros pueden ser cerrados lo cual indica que es para cierto grupo de alumnos y además protegido por una contraseña. Para el profesor, es interesante el uso de esta plataforma ya que los alumnos tienen la oportunidad de hablar y esto permite un apoyo más individualizado.

Al grabar un video se manifiesta el contexto social en el que se realiza y aporta elementos importantes de la identidad de quien se está grabando, desde su apariencia física y presentación, así como el espacio personal tal como el ambiente de su casa, oficina, la ciudad, el área educativa, u objetos que rodean a la persona, muebles, música o sonidos presentes, los cuales muestran aspectos de la personalidad. Una vez grabado el vídeo, la aplicación permite realizar una fotografía que se muestra como portada (Concheiro, Espejel y Pujolà ,2021).

Flipgrid se puede usar en todo tipo de dispositivos (PC, Laptop, tableta y smartphone), desde su sitio web como desde la aplicación móvil (disponible para Android e iOs) (Gosse, 2019).

\subsection{Uso de Flipgrid en la Educación}

Green y Green (2018) menciona que los debates en línea son una herramienta pedagógica efectiva ya que fomentan el pensamiento crítico, sin embargo, en los debates textuales faltan la relación personal que supone de ver y escuchar a la persona con la que se está hablando. Una herramienta que se utiliza con los estudiantes para fomentar la relación personal durante los debates en línea con videos cortos es flipgrid, con está los estudiantes podrán verse y oírse mutuamente.

Concheiro, Espejel y Pujolà (2021) mencionan que en los entornos digitales la manera de expresarse y trasmitir las emociones es diferente según el canal que se utilice. En el chat, se ha creado novedosas maneras de expresar el sentido del humor y gustos mediante emojis, stickers. En canales audiovisuales, la imagen, los gestos, las fotografías, el uso de avatares son formas de hacer que las conversaciones digitales sean más próximas y cálidas.

Gosse (2019) afirma que la herramienta de Flipgrid ha sido utilizada en Vechtdal College, Ámsterdam, en donde se enseña la materia de español para fomentar la expresión oral al aprender otro idioma y mejorar la confianza del alumno, se pueden grabar monólogos o diálogos. El feedback puede ser por escrito o de forma oral, grabando otro vídeo. Una ventaja para el profesor es que los vídeos entregados se ven en un solo sitio, además, el profesor controla la duración del vídeo que se va a entregar. Una desventaja es el tiempo que se necesita para escuchar a todos los alumnos y comentar todos sus vídeos.

De acuerdo con Concheiro, Espejel y Pujolà (2021) en los intercambios virtuales, el primer contacto entre los estudiantes determina la manera en la que se relacionarán durante el proceso educativo. Se realizó una actividad inicial en un proyecto de telecolaboración entre profesores de la Universidad de Barcelona y estudiantes que asisten a la Universidad de Islandia en un intercambio virtual, los profesores graban un video de presentación utilizando la herramienta de Flipgrid invitando a los estudiantes a elegirlos, así como los estudiantes responden con un video a tres profesores, está actividad contribuyó a generar interés y disminuye la ansiedad al conocer a sus compañeros previamente.

En el contexto actual por la pandemia Covid-19 en el Instituto Tecnológico de Estudios Superiores de Monterrey, CCM y en la Escuela Nacional de Lenguas, Literatura y Traducción (ENALLT-UNAM) en el curso de Estrategias educativas utilizaron la aplicación 
de Flipgrid para evaluar la comunicación en los alumnos, relatando historias (Storytelling) quienes prepararon videos sobre un tema que incluyen contenidos de distintas culturas ,buscando información, además de intercambiar ideas y permite ver si el estudiante es espontaneo en tiempo real (Gil,2020).

Flipgrid ha sido utilizada en la Universidad César Vallego, Perú en la materia de matemáticas para explicar problemas de algebra, los estudiantes tuvieron que exponer la demostración de expresiones en pocos minutos, esta tecnología fomenta el aprendizaje social pues los estudiantes pueden compartir y comentar sus opiniones sobre el trabajo de otro compañero (Sánchez, 2020).

Sanabria (2020) realizó un proyecto para fomentar competencias en alumnos de la Universidad de Costa Rica, se les solicitó crear un producto o servicio tecnológico nuevo, que no exista actualmente y presentarlo a través de un video realizado por ellos en la plataforma filpgrid grabado con su computadora $\mathrm{o}$ teléfono celular, para el cual debían investigar las tendencias actuales y avances tecnológicos.

Ramos (2020) implementó el uso de la aplicación de Flipgrid para mejorar la comunicación y elaborar actividades colaborativas en una escuela secundaria pública de Shuid, Cantón Alausí, con ella los alumnos con un nivel de inglés Pre-A1. realizaron sus tareas, grabaron sus videos, comentaron las tareas de sus compañeros y evaluaron su trabajo usando una rúbrica.

(Keiper et al., 2020) realizó un estudio para conocer las percepciones de los estudiantes sobre los beneficios de Flipgrid en los ambientes híbridos (HyFlex), los resultados obtenidos muestran que los estudiantes encuentran útil y benéfico el uso de Flipgrid en ambientes de aprendizaje híbridos. A si mismo (Lowenthal \& Moore, 2020) obtuvieron similares resultados, pero aplicando Flipgrid en cursos en línea. De igual manera (Saidi et al., 2021) investigo las preferencias de profesores y estudiantes sobre los sistemas de gestión del aprendizaje (SGA), como resultados de su estudio: Google classroom, SGA institucional, Schoology,
Edmodo y Flipgrid son los SGA que reportaron una mayor preferencia.

En el trabajo de (de Tantillo \& Christopher, 2020) proponen una serie de buenas prácticas para la adaptación de la formación de postgrado en enfermería en respuesta al reto actual que supone la pandemia de COVID-19 y las expectativas del distanciamiento social. Proponen herramientas que permitan los mensajes de discusión en vídeo asíncrono (canvas y flipgrid), permitiendo a profesores y estudiantes mantenerse socialmente conectados.

\section{MÉTODO}

El alcance definido para este estudio es del tipo exploratorio descriptivo, con enfoque de tipo mixto. En acuerdo con HernándezSampieri \& Mendoza Torres (2018), el tipo descriptivo se utilizó para describir los elementos importantes del tema en estudio y el tipo exploratorio para la investigación que corresponde. La investigación consiste en reportar una experiencia innovadora en el proceso de enseñanza aprendizaje y como medio de interacción durante la pandemia COVID-19 en estudiantes de la carrera de Tecnologías de Información de una universidad del noreste de México. El objetivo fue que los estudiantes utilizaran la herramienta de flipgrid como un recurso de las TIC a través del cual se puede generar conocimiento y competencias en los estudiantes, de estas últimas tanto técnicas como blandas.

El tipo de la muestra fue no probabilístico o por conveniencia, en el cual participaron 53 estudiantes que cursaron la materia de programación y base de datos en primer y segundo semestre respectivamente, durante el periodo febrero - junio del 2021 de la carrera de Licenciatura en Tecnologías de la Información en una universidad del noreste de México. La muestra es importante ya que los estudiantes no han podido llevar sus clases de forma presencial y no habían tenido la oportunidad de convivir o conocer a sus compañeros, interactuando en un ambiente de aprendizaje y de relación social. 
El enfoque mixto se definió para aplicar un trabajo conjunto con datos cualitativos y cuantitativos que permitan determinar áreas de oportunidad y enriquecer las estrategias de enseñanza aprendizaje (ver Figura 1).

Respecto a la parte cuantitativa se diseñó y aplicó un instrumento de tipo encuesta integrada por 8 preguntas cerradas categorizadas en una escala de Likert de 5 puntos. La obtención de los datos se llevó a cabo a través de la herramienta Microsoft Forms, de la cual se descargó un archivo en formato Microsoft Excel, mismo que se analizó en la herramienta de programación $\mathrm{R}$ Studio para determinar el grado de acuerdo en relación con las respuestas obtenidas, para esto se aplicó el modelo de consenso de Tastle (2006). En la prueba de confiabilidad del instrumento se obtuvo un valor de 0.93 , considerándolo como excelente.

Para el enfoque cualitativo, se aplicó una metodología sistémica la cual consistió en cinco etapas: Planeación, desarrollo, obtención de los datos, análisis de los datos y evaluación. En la etapa de planeación se definieron y desarrollaron dos actividades, en la primera se les solicitó a los estudiantes hacer uso de la herramienta Flipgrid para que conocieran a sus compañeros de clase e interactuaran de una forma asíncrona a través de la grabación de videos cortos; esta actividad tuvo como objetivo el generar habilidades de interacción social y de comunicación. En la segunda actividad se solicitó el desarrollo de una actividad técnica en relación a cada una de las materias, para el desarrollo debían grabar con la herramienta Flipgrid un video corto mostrando su cámara y pantalla; el objetivo de esta actividad fue que el estudiante genere conocimiento, aprenda a ser autodidacta, a que sea más original en sus actividades y a generar entre ellos la enseñanza, ya que podían ver los videos desarrollados por sus compañeros. En la etapa de desarrollo se definen las instrucciones detalladas de la actividad a realizar, los criterios de evaluación y los tiempos de entrega. En las etapas de obtención de los datos se desarrollan y aplican los instrumentos a utilizar en este estudio (encuesta e interacción en el aula con el uso de la herramienta Mentimeter). En la etapa de análisis de los datos y evaluación, se obtienen los resultados del instrumento aplicado y del cual ya se habló en esta sección; los videos fueron evaluados aplicando una rúbrica y se realiza un análisis y obtención de información sobre la experiencia desarrollada en una reunión virtual.

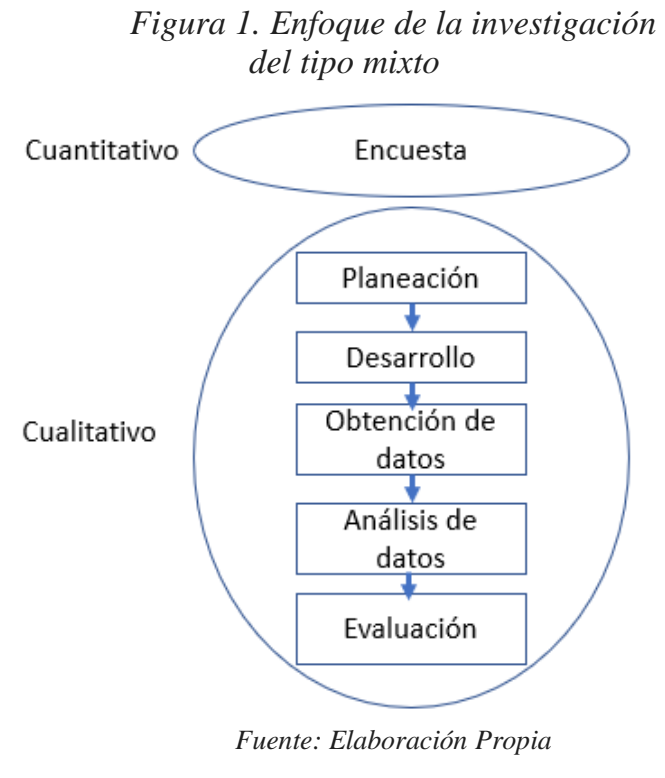

\section{RESULTADOS}

De la recolección y análisis de datos, se obtiene que de los estudiantes que participaron en la investigación el 28.3\% son del género femenino y el $71.7 \%$ son del género masculino; sus edades están en un rango de 17 a 23 años.

Figura 2. Genero de los estudiantes 


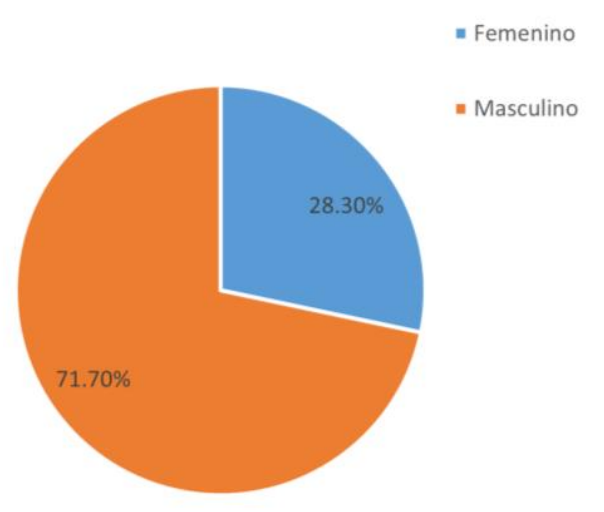

Fuente: Elaboración propia

Para el desarrollo de la actividad el $96.23 \%$ de los estudiantes utilizaron una computadora personal y el $3.77 \%$ utilizó un Smartphone.

Figura 3. Tipo de dispositivo utilizado por los estudiantes

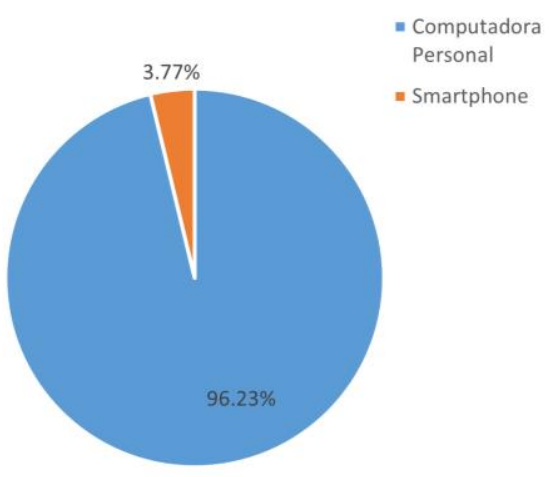

Fuente: Elaboración propia

El $71 \%$ de los participantes están totalmente de acuerdo en que la herramienta al permitirles ver los videos de sus compañeros, les ayudo a entender cómo realizar su actividad. Esto permite que los estudiantes aprendan enseñando a otros y se conviertan en creadores de contenidos. videos de sus compañeros

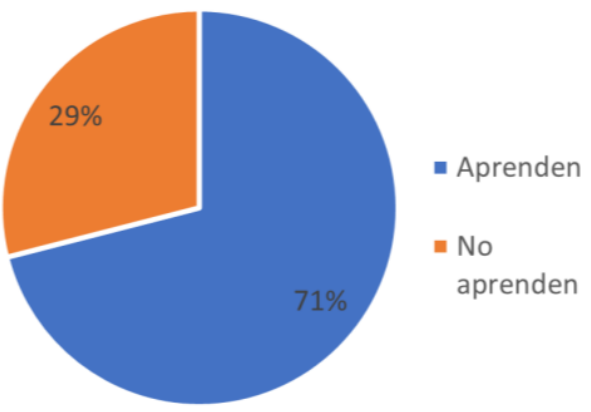

Fuente: Elaboración propia

El $70 \%$ de los participantes están totalmente de acuerdo en que la herramienta les facilitó la asimilación de algunos conceptos o elementos del tema expuesto.

Figura 5. A los estudiantes se les facilitó la asimilación de conceptos o elementos del tema

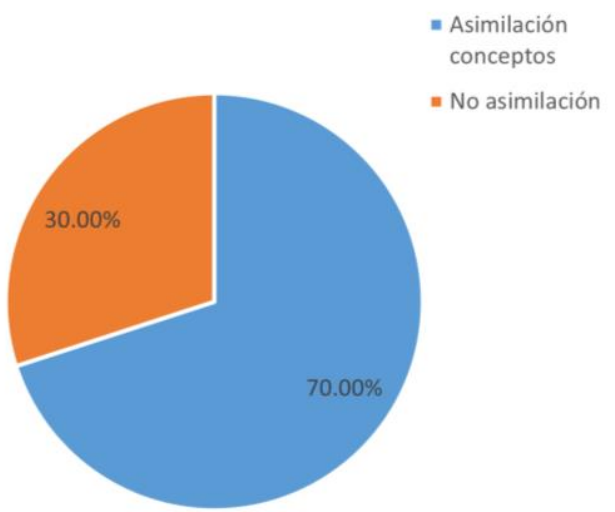

Fuente: Elaboración propia

El $65 \%$ de los estudiantes están totalmente de acuerdo en que su rendimiento $\mathrm{y}$ aprendizaje sobre un tema puede mejorar con el uso de la herramienta.

Figura 6. Estudiantes que mejoran su rendimiento y aprendizaje con el uso de la herramienta

Figura 4. Estudiantes que aprenden de los 


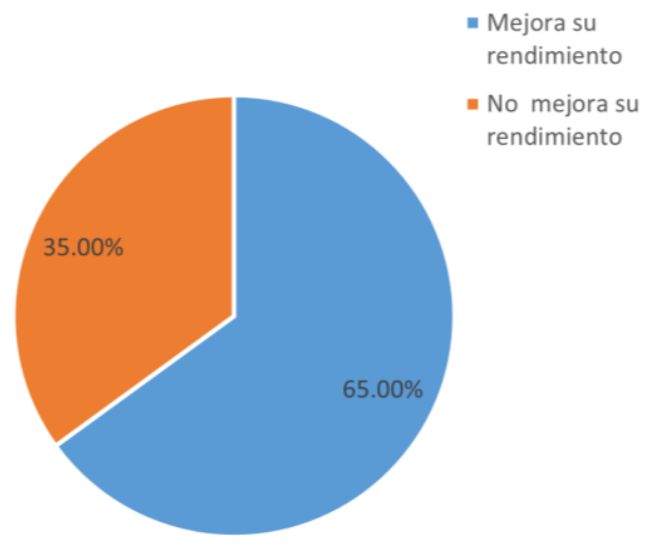

Fuente: Elaboración propia

El $67 \%$ de los participantes están totalmente de acuerdo que con el uso de la herramienta le permitirá ampliar su habilidad en comunicación o expresión oral.

\section{Figura 7. Mejoran su habilidad de comunicación o expresión oral}

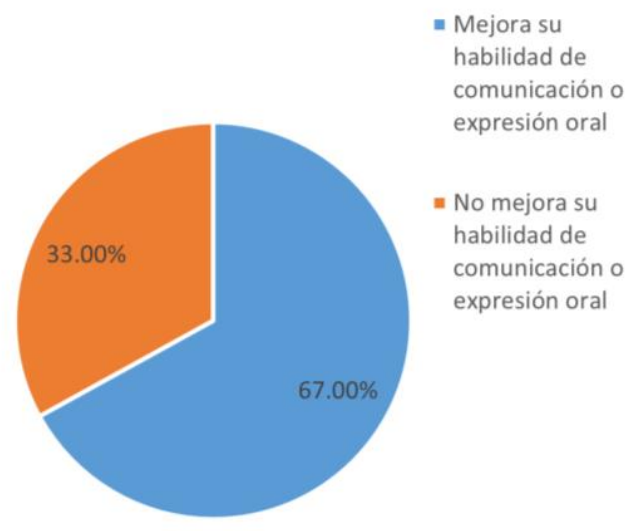

Fuente: Elaboración propia

El $68 \%$ de los estudiantes están totalmente de acuerdo en que les gustaría utilizar en el futuro la herramienta si tuvieran la oportunidad de hacerlo.
Figura 8. Les gustaría utilizar la herramienta en otra ocasión

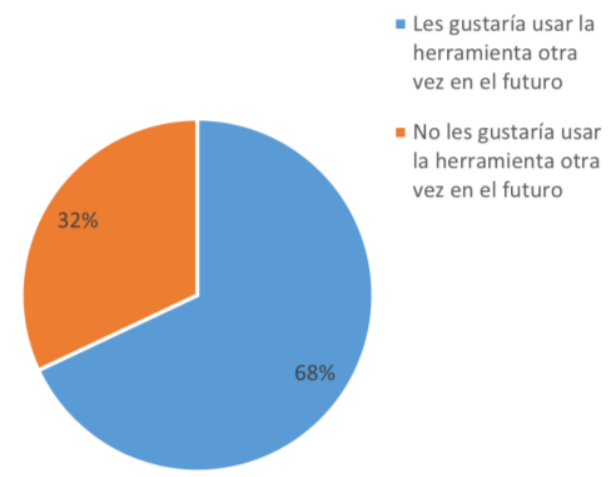

Fuente: Elaboración propia

El $67 \%$ de los participantes están totalmente de acuerdo en que les gustaría poder utilizar la herramienta para tareas de otras materias.

Figura 9. Les gustaría utilizar la herramienta en otras materias

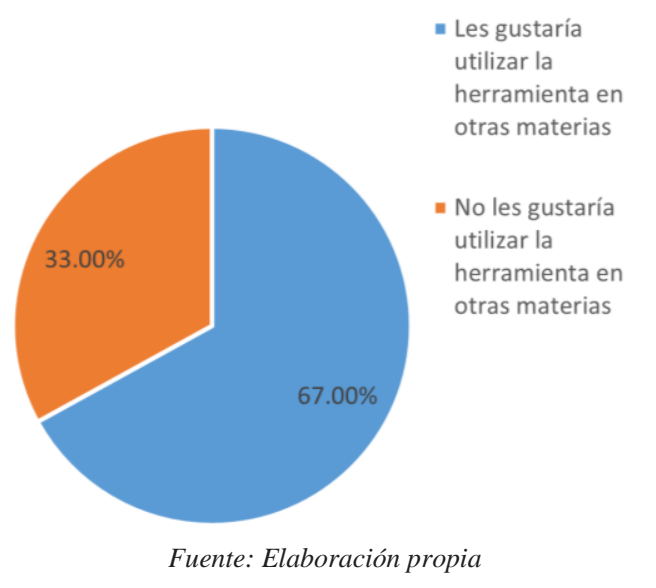

El $68 \%$ de los estudiantes están totalmente de acuerdo en que disfrutaron mucho de la herramienta porque les permitió conocer o ver a sus compañeros. 
Figura 10. Estudiantes que disfrutaron el uso de la herramienta

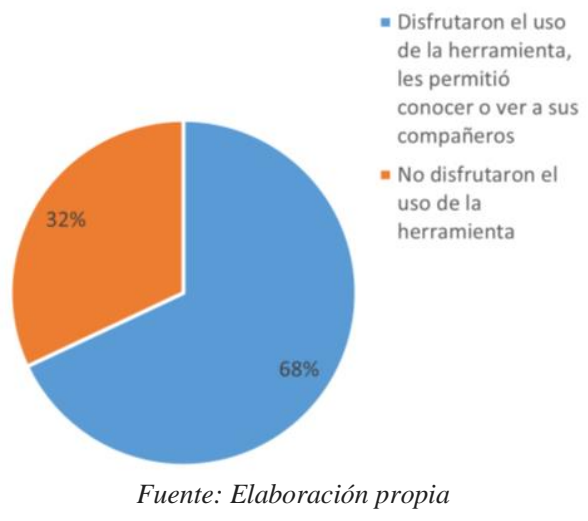

El $68 \%$ de los participantes están totalmente de acuerdo en que realmente fue un placer aprender el uso de la herramienta.

Figura 11. Fue placentero aprender a usar la herramienta

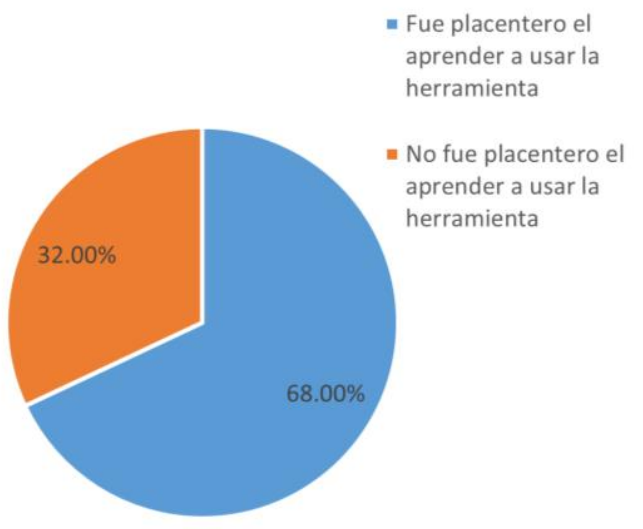

Fuente: Elaboración propia

Los problemas reportados con el uso de la herramienta pero que finalmente fueron resueltos por los estudiantes, indicaron que un $15 \%$ presentaron dificultad con la cámara, el 5\% manifestaron que fue muy poco el tiempo máximo que tendrían para el desarrollo del video, el 3\% señalaron haber tenido problemas con el rendimiento de su equipo de cómputo.

Figura 12. Fue placentero aprender a usar la herramienta

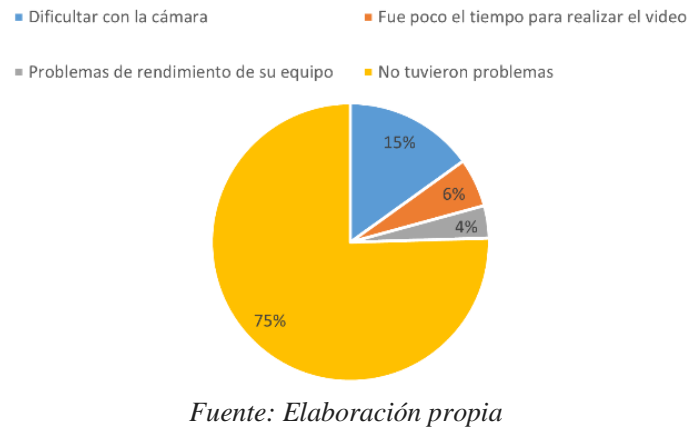

Cerca del 3\% indicaron haber utilizado alguna herramienta similar para el desarrollo de sus actividades académicas, pero esto fue durante su estancia en nivel medio superior.

Figura 13. Desarrollo de una actividad similar con la misma u otra herramienta.

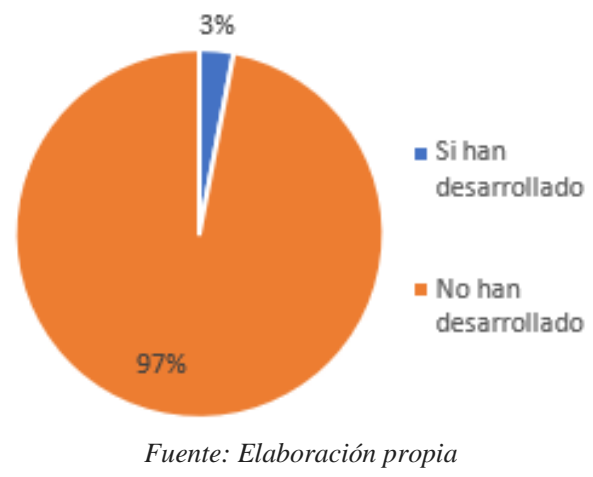

\section{CONCLUSIONES}

Los resultados ya presentados señalan un porcentaje promedio de satisfacción del $68 \%$ en el uso de la herramienta flipgrid, para el desarrollo de las actividades solicitadas a los estudiantes que participaron en este estudio. Del porcentaje faltante se puede considerar que hay estudiantes que no les gusta hablar y para algunos resulta más difícil el hablar frente a una cámara; aunado a lo anterior se presentan los nervios de que sus compañeros y maestro(a) del curso le conozcan. Por otro lado, al presentarse una actividad en la cual se requiere un desarrollo donde el estudiante debe preparar su narración para su video o el 
desarrollo de la práctica o actividad, implica una resistencia o aceptación a este método de enseñanza aprendizaje; además, es importante señalar que solo un 3\% refiere haber realizado alguna actividad académica de este tipo, solo que con un software diferente. Algo importante por mencionar es que la encuesta se aplicó al finalizar el semestre, lo cual pudo haber afectado en el porcentaje obtenido en la pregunta ¿si disfrutaron mucho de la herramienta porque les permitió conocer o ver a sus compañeros?, en la cual se obtuvo un $68 \%$ y esta fue la primera actividad del semestre.

Finalmente, considerando lo anterior, se hace necesario que el docente trate de ser creativo e innovador en el aula, buscando el generar el conocimiento y habilidades que los estudiantes requieren en su ámbito académico y profesional.

\section{REFERENCIAS}

Carrie Taylor, \& Timothy Hinchman. (2020). Strategies for Using Flipgrid in the Education. USChina Education Review B, 10(1). https://doi.org/10.17265/2161-6248/2020.01.003 
Concheiro, P., Espejel, O., \& Pujolà, J. T. (2021). Flipgrid: una app de vídeo para o intercambio virtual, a propinciudad y el aprendizaje de lenguas. Perspectiva, 39(1), 1-17.

de Tantillo, L., \& Christopher, R. (2020). Transforming graduate nursing education during an era of social distancing: Tools from the field. Nurse Education Today, 92(May). https://doi.org/10.1016/j.nedt.2020.104472

Gil, C. (2020). Storytelling, una estrategia para un aprendizaje significativo mediante la comunicación tecnológica. Revista Lengua y Cultura, 2(3), 13-21.

Green, T. y Green, J. (2018). Flipgrid: agregar voz y video a discusiones en línea. TechTrends , $62(1), 128-130$.

Gosse, L. (2019). El uso de FLIPGRID para fomentar la expresión oral. Mosaico. Revista para la promoción y apoyo a la enseñanza del español, (37), 31-42.

Keiper, M. C., White, A., Carlson, C. D., \& Lupinek, J. M. (2020). Student perceptions on the benefits of Flipgrid in a HyFlex learning environment. Journal of Education for Business, 0(0), 1-9. https://doi.org/10.1080/08832323.2020.1832431

Lowenthal, P. R., \& Moore, R. L. (2020). Exploring student perceptions of flipgrid in online courses. Online Learning Journal, 24(4), 28-41. https://doi.org/10.24059/olj.v24i4.2335

Megiddo, E. (2018). Microsoft y flipgrid se unen para llevar el aprendizaje social a estudiantes de todo el mundo. Microsof. Recuperado de: https://news.microsoft.com/eses/2018/06/18/microsoft-y-flipgrid-se-unen-para-llevar-el-aprendizaje-social-aestudiantes-de-todo-el-mundo/ [08/03/2021]

Mendoza, A. y Vázquez, V. (2020). REA para el aprendizaje a distancia que promuevan la motivación y la autonomía. Revista Lengua y Cultura, 2(3), 1-7.

Miao, F. Mishra, S \& Mc Greal, R. (2016) Open Educational Resources: Policy, Cost and Transformation. UNESCO and Commonwealth of Learning. Francia. https://unesdoc.unesco.org/ark:/48223/pf0000244365

Murray, S. (2018). Flipgrid: A modern tech tool to practice the age old art of speaking. Teaching \& Learning, Technology for Learning. Vol. 2 No. 3. Retrieved from https://journal.canadianschoollibraries.ca/flipgrid-amodern-tech-tool-to-practicethe-ageold-art-of-speaking/

Ramos Cuadrado, C. M. (2020). Improve Oral Interaction through Collaborative Activities (Master's thesis, Universidad Casa Grande. Departamento de Posgrado).

Saidi, R. M., Sharip, A. A., Abd Rahim, N. Z., Zulkifli, Z. A., \& Md Zain, S. M. (2021). Evaluating Students' Preferences of Open and Distance Learning (ODL) Tools. Procedia Computer Science, 179(2019), 955-961. https://doi.org/10.1016/j.procs.2021.01.085

Stoszkowski, J. (2018). Using Flipgrid to develop social learning. Compass: Journal of Learning and Teaching, 11(2). https://doi.org/10.21100/compass.v11i2.786

Sanabria, E. (2020). Docencia estratégica para fomentar las habilidades blandas en estudiantes de Informática Empresarial: experimento didáctico en el Recinto de Paraíso de la Universidad de Costa Rica. Technology Inside by CPIC, (6),1-21.

Sanchez, C. I. (2020). Herramientas tecnológicas en la enseñanza de las matemáticas durante la pandemia COVID-19. HAMUT'AY, 7(2), 46-57.

Silva, J. (2017). Un modelo pedagógico virtual centrado en las E-actividades. REd. Revista de Educación a distancia, 53(10), 1-20. doi:10.6018/red/53/10

Tastle, W. J., \& Wierman, M. J. (2007). Consensus and dissention: A measure of ordinal dispersion. International Journal of Approximate Reasoning, 45(3), 531-545.

Walss, M.E. (2021). Diez herramientas digitales para facilitar la evaluación formativa. Revista Tecnología, Ciencia y Educación, (18), 127-139. 